\title{
Strates
}

STRATES Matériaux pour la recherche en sciences sociales

$12 \mid 2006$

Nouvelles tensions impériales et recompositions en

Europe centrale, orientale, et CEI

\section{L'impact du programme Tacis dans les années 1990 : une tentative d'appréciation}

\section{Laure DELCOUR}

\section{OpenEdition}

Journals

Édition électronique

URL : http://journals.openedition.org/strates/2132

DOI : $10.4000 /$ strates.2132

ISSN : $1777-5442$

Éditeur

Laboratoire Ladyss

Édition imprimée

Date de publication : 31 décembre 2006

ISSN : 0768-8067

Référence électronique

Laure DELCOUR, «L'impact du programme Tacis dans les années 1990 : une tentative

d'appréciation », Strates [En ligne], 12 | 2006, mis en ligne le 20 juillet 2007, consulté le 08 septembre 2020. URL : http://journals.openedition.org/strates/2132 ; DOI : https://doi.org/10.4000/strates.2132

Ce document a été généré automatiquement le 8 septembre 2020.

Tous droits réservés 


\title{
L'impact du programme Tacis dans les années 1990 : une tentative d'appréciation
}

\author{
Laure DELCOUR
}

1 En 1991, la Communauté européenne décide de mettre en place un programme d'assistance technique pour soutenir l'URSS dans ses réformes politiques et économiques. Redéployé sur une base nationale après l'effondrement de celle-ci, ce programme baptisé Tacis s'impose au cours des années 1990 comme le principal levier de l'aide internationale aux anciennes Républiques soviétiques.

2 Comment apprécier l'impact de l'aide européenne sur la recomposition de l'espace post-soviétique?

3 On se limitera ici au cas de la Russie, qui a bénéficié de près de la moitié des fonds du programme Tacis ${ }^{1}$; c'est aussi dans ce pays que l'Union européenne (UE) a utilisé la plus large panoplie d'instruments d'assistance.

4 Ce travail tentera d'analyser l'impact de Tacis en mettant successivement en lumière trois composantes de son action :

5 - l'impact de Tacis comme programme d'aide, c'est-à-dire la pertinence du type d'assistance choisi et des instruments utilisés ;

6 - l'efficacité de Tacis comme politique publique, à savoir la gestion du programme ;

7 - l'influence de Tacis en tant qu'instrument de politique étrangère, ou la définition de sa place dans les relations entre l'UE et la Russie.

L'impact de Tacis sur les réformes russes

8 Le lancement de programmes d'assistance pour les pays d'Europe centrale, puis d'exURSS, confronte la Communauté européenne à des défis inédits. La transition économique et politique à laquelle se préparent les anciens pays communistes est une «première » : ces États ne peuvent s'appuyer sur aucune référence historique pour choisir une stratégie de réformes. D'autre part, pour la première fois aussi, les 
bénéficiaires de l'aide ne sont pas des pays en voie de développement, mais des « Second World countries ${ }^{2}$ ». Ces spécificités exigent des bailleurs de fonds un effort d'adaptation.

Or, même si les acquis de quarante années d'aide publique au développement ne sont que très partiellement utilisables, le programme Tacis s'inspire des modèles déjà mis en place pour d'autres régions. La nécessité de mettre en place très rapidement une aide en faveur de l'URSS explique l'absence de réflexion stratégique sur les méthodes et les principes de l'assistance. La Commission reproduit donc, dans la conception et la gestion de Tacis, des schémas déjà éprouvés ailleurs, en Afrique, Asie ou Europe de l'Est. La première similitude porte sur la nature de l'aide. L'assistance technique est adoptée par la Commission sans que d'autres possibilités soient réellement examinées. Or, aux yeux des Russes, cette forme d'aide est intrinsèquement liée aux pays en voie de développement. À la différence de ces derniers, la Russie dispose déjà des infrastructures nécessaires à sa transformation. Surtout, elle bénéficie d'un système éducatif performant et d'une élite intellectuelle et technique, donc d'un savoir-faire indéniable. Pour de nombreux Russes, le terme même d'assistance technique revêt une connotation négative ${ }^{3}$. L'assistance technique doit uniquement permettre à la Russie d'acquérir les compétences et de développer les infrastructures propres à l'économie de marché et à un système politique démocratique. Elle doit jouer un rôle d'appoint dans l'aide internationale, en aucun cas de pilier. Cette vision critique de l'assistance technique, fondée sur la volonté de distinguer la Russie des autres pays récipiendaires de ce type d'aide, est bien résumée par un expert russe de l'unité de coordination Tacis :

Nous n'avons pas besoin d'apprendre à construire des fusées, nous avons besoin qu'on nous dise comment les construire dans un contexte de manque de financement $t^{4}$.

10 Ces critiques sont encore accentuées par l'absence de résultats immédiats des programmes d'assistance technique. Ceux-ci reposent sur la transmission d'un savoirfaire via des instruments tels que le policy advice, les voyages d'étude ou la formation. De tels instruments sont, en soi, difficiles à évaluer. En particulier, les résultats à long terme des projets, c'est-à-dire leur impact, sont à la fois diffus et étalés dans le temps. Ainsi, un projet dans le secteur météorologique a des résultats directement visibles - la constitution d'un recueil de données, de méthodes d'observation... - , mais, à plus long terme, ces données vont avoir un impact sur d'autres secteurs (par exemple l'agriculture) qu'il sera impossible de quantifier, et dont l'origine même sera difficilement décelable ${ }^{5}$. L'impact indirect et qualitatif de l'assistance technique explique que le programme Tacis soit quelquefois perçu par les Russes comme un simple outil économique et politique au service des intérêts européens.

11 Le sentiment d'un transfert vers l'ex-URSS d'un modèle d'assistance créé pour les pays ACP (Afrique, Caraïbes, Pacifique) est lié également au manque de spécialistes de la Russie au sein de la Commission ${ }^{6}$. Une telle carence s'explique par l'inexistence de relations officielles entre l'URSS et la Communauté, de la création de celle-ci à la fin des années 1980, et par la politique de la Commission en matière de ressources humaines après cette date. Pour gérer le programme Tacis, la Commission a fait appel à des agents temporaires et à des fonctionnaires affectés à d'autres directions générales, notamment à la DG VIII (Développement et Coopération avec l'Afrique, les pays Caraïbes et le Pacifique). Ces personnes sont devenues responsables de la gestion de Tacis sans y être réellement préparées : au cours des années 1990, la Commission n'a pas mis en place de politique de formation en matière de gestion des aides extérieures ${ }^{7}$. 
La méconnaissance de la Russie régnant au sein des services de la Commission a été d'autant plus mal ressentie sur le terrain que les consultants responsables des projets Tacis avaient essentiellement, eux aussi, des "états de service» en Afrique ${ }^{8}$. Cette situation a été en partie corrigée à partir du milieu des années 1990, avec l'intégration dans les services Tacis à Bruxelles de quelques personnes « du terrain » (c'est-à-dire ayant une première expérience de gestion de projets Tacis en Russie) ou d'effectifs venant du programme Phare. Mais la réorganisation administrative de la Commission en 1998 gomme les spécialisations géographiques en unifiant la gestion des aides aux pays tiers au sein d'un organisme appelé SCR, puis EuropeAid; la majorité des effectifs des unités communes n'a qu'une expérience limitée de Tacis9.

La prépondérance d'acteurs spécialistes des pays ACP et l'imitation - au moins partielle - de schémas appliqués à l'aide au développement ${ }^{10}$ ont pesé sur les relations avec les bénéficiaires du programme: celui-ci est apparu inadapté aux yeux des Russes $^{11}$. La Commission a pourtant su adapter les priorités et les instruments de Tacis à l'évolution des réformes en Russie. Au début des années 1990, la sécurité alimentaire, la formation à la gestion et le soutien aux entreprises et aux services financiers sont les principaux objectifs du programme, au moment où la Russie doit faire face aux conséquences économiques de la dissolution de l'URSS et où elle lance un vaste chantier de réformes. Dès le milieu des années 1990, ces priorités sont réajustées pour tenir compte des conséquences de la transition: Tacis inclut alors la protection sociale et l'environnement au rang de ses priorités. À partir de la fin des années 1990, le programme met davantage l'accent sur le renforcement de l'État de droit et l'équilibre des pouvoirs entre centre et régions. La capacité d'adaptation de Tacis à l'environnement russe est encore démontrée par l'inclusion dans le programme, en 1996, d'un nouvel instrument : l'investissement, permettant de financer des projets d'infrastructures; dès lors, Tacis ne se limite plus à l'assistance technique et la part de l'investissement dans le programme va croissant ${ }^{12}$. Au fil des années, la Commission s'est ainsi efforcée de diversifier ses instruments et de concentrer son action sur quelques secteurs clés. Cet ajustement constant montre que l'aide européenne a eu un rôle d'accompagnement dans le processus de réformes russes, plus que d'initiation ou d'orientation.

Le programme Tacis comme politique publique : les déficiences de la gestion de l'aide L'impact de l'aide européenne a longtemps été limité par la gestion même de Tacis, extrêmement complexe: jusqu'à la réforme de 2000, la lourdeur des procédures combinée au manque de personnel a rendu l'assistance européenne inopérante.

La Commission européenne, chargée de la gestion de Tacis, a dû faire face au début des années 1990 à un accroissement de ses responsabilités en matière de coopération extérieure $^{13}$. Avec l'inclusion de l'ancien bloc communiste dans sa sphère d'action, la politique de coopération européenne acquiert véritablement un caractère universel. Mais ce processus d'universalisation s'est effectué de manière spontanée, par agrégation de nouveaux programmes reposant en partie sur les mécanismes historiques de la coopération européenne, en partie sur des instruments nouveaux. L'universalisation a ainsi rendu extrêmement complexe le système européen de coopération extérieure. Elle a eu pour résultats la multiplication des programmes, la prolifération des lignes budgétaires et des procédures de gestion et, in fine, le morcellement de la politique d'assistance. La gestion interne de chaque programme est également extrêmement fragmentée. Pour le seul programme Tacis, il existe, à la fin 
des années 1990, 21 sous-programmes, régis par des procédures différentes, et plusieurs lignes budgétaires.

Plus que les autres programmes de coopération extérieure, Tacis est affecté par l'éclatement des structures administratives de la Commission et les modifications du cadre institutionnel tout au long des années 1990. Le programme est intégré à la direction créée pour le programme Phare, de sa création jusqu'en 1993 ; l'assimilation de fait entre les pays d'Europe centrale et l'ex-URSS ne permet pas de prendre suffisamment en compte les spécificités de cette dernière, au moment où sont lancées les réformes les plus importantes en Russie. Par la suite, si Tacis s'émancipe de la direction Phare, les changements organisationnels intervenus au sein de la Commission posent de sérieux problèmes de coordination. De 1993 à 1995, Tacis est intégré à la DG I (Relations économiques extérieures) dirigée par Leon Brittan, alors que les relations politiques avec la Russie sont gérées par la DGIA dirigée par Hans van den Broek. La commission Santer (1995-1999) assouplit la division entre relations politiques et économiques, mais la structuration géographique retenue aboutit à une fragmentation des services: si elle renforce la cohérence entre programmes d'aide et stratégie politique dans un pays donné ${ }^{14}$, elle ne permet pas à la Communauté de développer une approche globale en matière de coopération extérieure ${ }^{15}$. La première tentative de réforme, en 1998, ne remporte pas le succès escompté. La création d'un Service Commun Relex (SCR) chargé de l'aide au développement devait permettre l'homogénéisation des procédures de gestion ${ }^{16}$. Elle introduit au contraire une nouvelle coupure dans le cycle de projets, entre les activités de programmation et d'identification, qui restent dévolues aux unités géographiques de la DGIA, et la mise en œuvre.

16 L'imbroglio administratif né de l'universalisation de la coopération européenne a d'autant plus pesé sur l'efficacité des programmes que la Commission manquait d'effectifs pour mettre en œuvre la politique d'aide. En effet, la croissance exponentielle du budget de coopération extérieure ne s'est pas accompagnée d'une augmentation proportionnelle des ressources humaines. En dix ans, le volume de l'assistance a été multiplié par 2,8 et les effectifs par 1,8 seulement. En 1999, 2000 personnes (fonctionnaires et agents auxiliaires) sont en charge des programmes de développement à Bruxelles pour un total de 30000 projets contractés; ce personnel doit également assurer l'interface avec les autres institutions communautaires. La Commission estime alors que le déficit en ressources humaines équivaut à 1300 postes. Dans le cas du programme Tacis, le manque d'effectifs a eu un impact particulièrement important, parce qu'il s'est fait sentir dès la création du programme, donnant lieu à des situations aberrantes. En 1991, la Commission a lancé Tacis en affectant à sa gestion un seul fonctionnaire et deux experts nationaux détachés. Cette pénurie de personnel a eu deux conséquences. Tout d'abord, une perte de qualité des projets. Ainsi, dans le cadre du programme Tacis à la fin des années 1990, les fonctionnaires de la Commission à Bruxelles géraient en moyenne 30 projets chacun ${ }^{17}$, à des stades différents et dans des régions dont ils ne possédaient pas forcément une connaissance approfondie.

Surtout, le manque de ressources humaines a entraîné une perte générale de contrôle de la Commission sur les programmes lancés. La pratique de la sous-traitance, courante pour la mise en œuvre des projets, s'est étendue à des tâches de gestion et d'identification avec la création de bureaux d'assistance technique. Dans la gestion de l'aide au développement, elle a eu deux conséquences. Elle a d'abord généré des 
dépenses bien supérieures au transfert de personnel, au recrutement d'agents temporaires ou même de fonctionnaires. Dans le cadre du fonctionnement de Tacis, les tâches d'identification des projets sont ainsi confiées à une unité de coordination, composée pour moitié de Russes travaillant pour le ministère de l'Économie et pour moitié d'experts européens choisis par appel d'offres. Le personnel de l'unité de monitorat, chargé du suivi et de l'évaluation des projets Tacis, est recruté de la même manière; de même que les effectifs des bureaux d'assistance technique présents dans les régions russes. Ces dépenses liées au fonctionnement du programme ont représenté entre $5 \%$ (au milieu des années quatre-vingt-dix) et $9 \%$ (à la fin des années quatrevingt-dix) des fonds totaux de Tacis; ainsi, près de 43 millions d'euros sont alloués en 1998 à «l'aide à la mise en œuvre du programme ${ }^{18}$ ». Deuxièmement, la pratique de la sous-traitance a donné lieu à des collusions d'intérêt dénoncées par la Cour des comptes - condamnations reprises par les États membres, qui, pourtant, refusaient d'un autre côté l'augmentation budgétaire qui aurait permis à la Commission de recruter. Ainsi, dans le cadre de Tacis, la Cour a notamment condamné le cumul, par certains experts, des tâches de préparation et de mise en œuvre des projets ${ }^{19}$. La pratique de la sous-traitance reflète ainsi l'absence "de frontière nette entre les 'acteurs publics' et les "acteurs privés" " au sein de la gouvernance européenne ${ }^{20}$. Dans le cas de la coopération extérieure, elle a considérablement contribué à décrédibiliser l'action de la Commission.

$\mathrm{Au}$ total, ces facteurs se sont conjugués pour rendre la politique de coopération extérieure inopérante. La Commission a accumulé des retards importants en amont du cycle de projet (programmation), répercutés en aval (signature des contrats et déboursements). Les retards accumulés conduisent à des arriérés de paiement; se sont ainsi développés les " engagements dormants ", dont le montant était de 1155000000 euros pour Tacis en 1999 - somme correspondant à trois années d'engagement de fonds et deux années de signature des contrats ${ }^{21}$ ! Pour Tacis, le délai moyen entre l'identification des projets et le début de la mise en œuvre est estimé à 18 mois-2 ans dans les années 1990. Dans un pays où la situation politique et économique évolue très rapidement, ce délai a eu un impact très négatif sur l'efficacité des projets : pour la grande majorité d'entre eux, les termes de référence décrivant les objectifs et tâches à accomplir ont dû être reformulés ; dans quelques cas plus rares, le bénéficiaire identifié pour un projet avait disparu au moment du début des travaux ${ }^{22} \ldots$

L'assistance comme outil de politique extérieure : l'influence de Tacis sur les relations UE-Russie

programme Tacis a eu un impact limité sur le processus de réformes, il a en revanche tenu depuis sa création une place fondamentale dans les relations entre la Russie et l'UE. En l'absence de stratégie politique européenne, la présence de l'Union dans l'ex-URSS s'est affirmée par le biais des programmes d'aide: Tacis a été utilisé comme un instrument de politique extérieure, pour façonner des liens avec la Russie.

Jusqu'au milieu des années 1990, l'UE a peiné à élaborer une vision d'ensemble pour sa politique en Russie. Au lendemain de l'effondrement de l'URSS, la volonté affichée par la Commission et le Conseil de conclure rapidement un nouvel accord avec la Russie ${ }^{23}$ dissimule de profondes hésitations sur le type de politique à mettre en place. L'accord avec la Russie est d'abord défini négativement, par opposition aux accords européens signés avec les pays d'Europe centrale et par distinction avec les autres ex-républiques soviétiques ${ }^{24}$. Ces tâtonnements s'expliquent par les divergences entre acteurs 
communautaires sur la politique à développer en Russie. Chargée de mener les négociations au nom de la Communauté, la Commission tente de concilier les vues du Parlement européen, favorable à un rapprochement très net avec Moscou, et celles des États membres qui cherchent à préserver leurs intérêts tout en se montrant prudents sur la politique à développer en Russie. L'accord de partenariat et de coopération, signé à Corfou le 24 juin 1994 après de longs mois de négociations, apparaît donc comme un compromis fragile ${ }^{25}$. Le régime commercial accordé à la Russie est un régime " hybride ${ }^{26}$ ", combinant des pratiques réservées habituellement aux pays à commerce d'État (évaluation de la valeur des prix en se référant à un pays tiers pour l'application de mesures antidumping), la libéralisation des échanges (démantèlement immédiat des restrictions quantitatives) et des dérogations à cette libéralisation (maintien de restrictions pour les produits textiles et sidérurgiques russes, possibilité de protection temporaire pour l'industrie russe). Les dispositions politiques (l'affirmation d'un socle de valeurs communes ${ }^{27}$ ) étant énoncées sous forme de principes, seule une analyse de leur mise en cuvre peut déterminer le caractère formel ou rigoureux de la conditionnalité, comme la portée réelle du dialogue politique prévu dans l'accord.

S'il propose un cadre aux relations russo-européennes, l'accord de partenariat, en raison de ses ambiguïtés, ne permet pas en soi de donner une impulsion nouvelle à ces relations. Dans ce contexte, l'UE affirme sa présence en Russie par le biais du programme d'aide géré par la Commission: Tacis devient au quotidien le pilier des relations russo-européennes.

Tacis s'efforce de développer les partenariats à tous les niveaux. Le caractère novateur de l'assistance technique communautaire réside notamment dans l'implication des régions russes. Dès 1991, des régions prioritaires sont désignées, qui formeront le cœur des actions d'assistance technique. La Communauté s'efforce de mettre en place dans ces zones des programmes cohérents, fondés sur un secteur prépondérant en fonction des atouts de la région bénéficiaire (ainsi l'agriculture pour la région de Samara, en 1992). La sélection des régions prioritaires obéit à plusieurs impératifs. La Communauté européenne choisit d'abord de cibler son action en direction des régions les plus avancées dans les réformes, ou dont l'administration est favorable aux réformes (par exemple Saint-Pétersbourg au début des années 1990). La sélection des régions pilotes obéit également à des motivations d'ordre économique : la désignation de Tyumen en 1992-1993, puis de la Sibérie occidentale, est dictée par la richesse en ressources naturelles de ces zones. Enfin, la position stratégique de certaines régions conduit la Communauté à les privilégier : c'est le cas pour Kaliningrad, et surtout pour la Russie du nord-ouest en 1995, au moment de l'entrée de la Finlande dans l'UE. Mais cette volonté explicite de prendre en compte les niveaux régional et local dans les actions d'assistance repose sur des motivations plus implicites. La Communauté espère ainsi développer des relations horizontales avec les régions russes sans obligatoirement passer par le centre. Miser sur les régions permet d'accroître l'efficacité de l'aide, mais aussi sa visibilité. Confrontée à l'incertitude dans ses relations avec le nouvel État russe, la Communauté voit dans le développement de liens horizontaux une opportunité de parvenir à ses objectifs déclarés - le renforcement de la démocratie et de l'économie de marché, tout en se conformant à ses propres intérêts et à ceux de ses acteurs (États membres ou agents économiques). Dès lors, la Commission crée des sous-programmes dont le but est de développer les liens entre certaines catégories d'acteurs européens et leurs homologues russes. Ces programmes s'efforcent de mettre en place un partenariat entre les sociétés civiles russe et européenne (ainsi le programme Démocratie) ${ }^{28}$, les 
entreprises $^{29}$, les organisations professionnelles ${ }^{30}$, les universités ${ }^{31}$ ou les administrations locales de Russie et des États membres de la Communauté.

Le programme d'assistance est donc implicitement utilisé comme un outil de politique étrangère, d'abord pour façonner une politique européenne en Russie, puis pour la mettre en œuvre. Tacis se voit attribuer un rôle clé dans la mise en œuvre des dispositions de l'accord de partenariat. Ce rôle revêt de fait une acception extrêmement large, en raison des ambiguïtés de l'accord et de la lenteur du processus de ratification. L'accord de partenariat entre en vigueur trois ans et demi après sa signature, le 1er décembre 1997. Dans l'intervalle, Tacis s'efforce d'intégrer les difficultés non prévues par l'accord ou de donner corps à certaines dispositions trop brèves. Ainsi, un projet d'un montant de cinq millions d'euros est lancé en faveur du département de droit public de l'administration présidentielle, impliqué avec le Parlement russe dans l'élaboration des lois ${ }^{32}$. Ce projet a pour but d'aider à renforcer la protection légale offerte aux opérateurs économiques. La filiation de ce projet avec l'accord de partenariat n'est pas explicite. Il s'inscrit dans la perspective de renforcement de la coopération économique entre la Russie et l'UE. À ce titre, on peut le rattacher à l'article 56 de l'accord, en vertu duquel les deux parties doivent développer leur coopération économique dans de nombreux domaines ${ }^{33}$. Pourtant, la protection des opérateurs économiques ne fait pas partie des thèmes dominants de l'accord. Si elle fait l'objet d'un projet d'assistance, c'est en raison du cours ultérieur des relations russocommunautaires et des transformations en Russie. Les premiers mois suivant l'entrée en vigueur de l'accord démontrent ainsi que les déficiences en matière de protection des opérateurs économiques constituent un obstacle au développement de la coopération russo-européenne. Rétrospectivement, la brièveté des dispositions de l'accord en la matière apparaît également préjudiciable au renforcement des liens. Ce projet Tacis s'efforce donc de combler le vide laissé par l'accord de partenariat, d'en pallier les insuffisances plutôt que d'en appliquer les dispositions stricto sensu.

La perspective de l'adhésion de dix nouveaux pays et la crise économique russe d'août 1998 conduisent l'UE à formaliser une stratégie politique à l'égard de la Russie. Cette stratégie commune, adoptée en juin 1999, s'attache à renforcer la cohérence entre les instruments utilisés parl'UE. Dans cette politique européenne renouvelée, le programme Tacis continue de jouer un rôle clé. Son insertion dans la stratégie de partenariat - à laquelle il donne chair - lui confère une dimension explicitement politique. Ainsi, fin 1999, c'est au programme Tacis qu'échoit l'application la plus directe de la conditionnalité dans la réaction européenne à l'intervention en Tchétchénie; le Conseil des Affaires générales réuni le 24 janvier 2000 décide de réorienter les projets Tacis vers le renforcement de la démocratie en Russie, mettant entre parenthèses les autres secteurs prioritaires ${ }^{34}$. Le programme Tacis joue ainsi une nouvelle fois un rôle d'application immédiate de la politique européenne en Russie.

L'insertion de Tacis dans une stratégie politique européenne souligne son apport fondamental et l'évolution de son rôle. La finalité essentielle de l'aide n'est plus, comme au début des années 1990, de transmettre un savoir-faire lié au fonctionnement de la démocratie et de l'économie de marché - quelles que puissent être les carences de la Russie dans ce domaine.

Depuis la fin des années 1990, l'impact de Tacis se mesure davantage en termes de transfert de règles et d'harmonisation de la législation et des normes russes avec celles de l'Union. La décision d'instaurer quatre espaces communs (espaces économique ; de 
sécurité extérieure ; de liberté, de sécurité et de justice ; de recherche et d'éducation), prise au sommet russo-européen de Saint-Pétersbourg en mai 2003, renforce ce rôle de relais normatif.

Ce lien entre assistance et stratégie politique est entériné dans la réforme de la gestion de l'aide européenne, entamée en 2000, qui confie un rôle-clé aux délégations de la Commission sur le terrain. Plus encore, l'élargissement de l'Union aux pays d'Europe centrale, en suscitant dès 2003 le lancement d'une politique de voisinage visant à renforcer les relations avec les États frontaliers de l'ex-URSS et de la Méditerranée, a joué dans le sens d'une politisation croissante du programme Tacis. Ainsi, l'adaptation du cadre législatif à celui de l'Union européenne, nécessaire à l'accès au marché unique prévu par la politique de voisinage, est désormais le cœur du programme Tacis qui, pour appuyer ce processus, a repris des instruments issus de l'élargissement comme les jumelages. Bien que n'étant pas pleinement concernée par la politique de voisinage, la Russie est partie prenant de cette évolution: la réalisation des quatre espaces communs, exigeant l'adaptation de la législation russe, conduit également à la politisation de Tacis en Russie. C'est donc désormais comme outil du rapprochement russo-européen et de politique extérieure qu'il faut analyser et évaluer le programme Tacis.

\section{NOTES}

1. [Ce texte a été présenté lors d'un séminaire à l'université Paris-X en avril 2002.]La Russie a reçu en dix ans 1329,55 millions d'euros sur un total de 2 916,01 pour Tacis soit près de $45 \%$. Elle a également bénéficié d'une partie des programmes régionaux et des actions d'appui à la mise en œuvre de Tacis ; mais les sources statistiques de la Commission européenne ne disent pas dans quelle mesure. Il est donc difficile de chiffrer le montant exact reçu par la Russie. Cf. Financial statistics, http://europa.eu.int/ comm/europeaid (consulté en 08/2006)..

2. Burnell P., Foreign Aid in a changing World, Buckingham/Philadelphie, Open University Press, 1997, p. 212.

3. D'autant plus qu'au cours des premières années, il était fréquemment traduit en russe par pomochtch [aide], et non sodejstvie [assistance] ou encore sotrudnitchestvo [coopération]. Cf. par exemple Evropejskie soobtchestva : nautchno-tekhnitcheskie programmy i tekhnitcheskaâ pomochtch Rossii [Les Communautés européennes : les programmes scientifiques et techniques et l'aide technique à la Russie], Moscou, Centre de coopération avec les Communautés européennes/Délégation de la Commission européenne, 1992, $185 \mathrm{p}$.

4. Entretien avec un expert russe de l'unité de coordination, Moscou, juin 1997.

5. Cf. R. Cassen, How Aid Works, p. 146-147.

6. De manière générale, ce manque de spécialistes est plus marqué dans l'aide communautaire que dans les programmes bilatéraux des États membres. Une étude comparative, menée à la fin des années 1990 sur les politiques d'assistance gérées par la Commission (DG VIII) et cinq États membres, conclut à une carence en personnel plus 
qualitative que quantitative au sein de la Commission. Cf. A. Cox, J. Healey, A. Koning, How European Aid Works. A Comparison of Management Systems and Effectiveness. Londres : Overseas Development Institute, 1997, 246 p.

7. La première réflexion d'ensemble sur la politique de formation des effectifs des services extérieurs date de la fin des années 1990. Cf. SEC (97) 605 du 8/04/1997 et COM (1999) 180 du 21/04/1999.

8. Cf. le discours d'un expert européen, interrogé à Moscou en mai 1998 : « Au début, c'était surtout des experts qui avaient travaillé en Afrique qui arrivaient et qui faisaient un petit exposé d'un quart d'heure sur l'assistance aux Russes, sans savoir à qui ils parlaient. En gros, les Russes avaient l'impression que seul le climat était différent. » 9. Entretien avec un fonctionnaire chargé de la préparation des appels d'offres, unité E1, S.C.R., Commission européenne, Bruxelles, janvier 2000.

10. Voir à ce sujet l'article de C. Ners, «L'assistance occidentale à la transition dans les pays postcommunistes ", Économies et sociétés, avril-mai 1992, p. 347-363.

11. Pour une vision russe critique de l'assistance occidentale, voir l'exposé paru dans Znamâ sous la direction de S. Karaganov, « Zapadnaâ pomochtch Rossii : v tchem eë ochibki ?» [L'aide occidentale à la Russie : en quoi est-elle erronée], Institut d'Europe de l'Académie des Sciences de Russie, avril 1994, p. 157-183.

12. Il est autorisé à hauteur de $10 \%$ du budget total du programme par le règlement de 1996 et de $15 \%$ par le règlement de 1999. Cf. Conseil de ministres de l'UE, règlement $n^{\circ}$ 1279/96 du 25 juin 1996 et règlement n 99/2000 du 29 décembre 1999.

13. Cf. F. Petiteville, « La coopération économique de l'UE entre globalisation et politisation ", Revue française de science politique, juin 2001, p. 452.

14. Ainsi, au sein de la DG IA, l'unité C2 est en charge à la fois des relations avec la Russie, de la programmation et de la mise en œuvre de Tacis.

15. La Commission Santer ne compte pas moins de cinq directions générales consacrées aux relations extérieures.

16. Commission européenne, Organization Chart of the Joint Relex Service for the Management of Community Aid to Non-Member Countries, janvier 1999.

17. Entretien avec un fonctionnaire de la DG Relex, Commission européenne, janvier 2000.

18. Commission européenne, DGIA, Rapport annuel Tacis 1998, COM (99) 380 final, Bruxelles, 23/07/1999, p. 52.

19. Cf. par exemple le point 12.31 du rapport relatif à l'exercice 1993 et le point $12.48 \mathrm{du}$ rapport relatif à l'exercice 1995. Les rapports annuels de la Cour des comptes sont disponibles sur: http://www.ccomptes.fr/Cour-des-Comptes/publications/rapports/ cdc55.htm (consulté en 04/06).

20. Cf. Ch. Lequesne, « Comment penser l'Union européenne? », in Smouts M.-C., Les nouvelles relations internationales, Paris, Presses de Sciences Po, 1998, p. 120-121.

21. Commission européenne, Rapport annuel Tacis 1999, COM (2000)

835 final, 20/12/2000.

22. Exemple cité par la Cour des comptes dans son rapport relatif à l'exercice 1992.

23. Destiné à remplacer l'accord de commerce conclu avec l'URSS en 1989.

24. La Commission établit une double différenciation entre, d'une part, l'Europe centrale et la Russie ; et d'autre part, entre cette dernière et les autres ex-républiques soviétiques. C. Hillion parle de twofold differentiation. C. Hillion, « Partnership and Cooperation Agreements between the EU and NIS of the ex Soviet Union », European Foreign Affairs Review, n³, 1998, p. 403. 
25. Proposition de décision de la Commission et du Conseil, COM (94) 257 final, Bruxelles, 15 juin 1994.

26. J. Lebullenger, « Un Accord de partenariat confronté aux règles du GATT et de l'OMC ", in J. Raux, V. Korovkin, Le partenariat entre l'Union européenne et la Fédération de Russie, Rennes, Apogée, 1998, p. 203.

27. L'article 2 de l'accord se réfère notamment à l'Acte final d'Helsinki et à la Charte de Paris pour une nouvelle Europe.

28. La description que fait la Commission des objectifs de Démocratie est particulièrement intéressante : «Ce programme est conçu pour répondre à un double objectif. Le premier consiste à donner à la Commission un outil pour ses relations extérieures, en renforçant l'émergence dans les nouveaux États indépendants d'une société pluraliste. Le second est de répondre à un large éventail de groupes d'intérêt, d'associations et d'organisations non-gouvernementales qui œuvrent au développement d'une société favorisant le pluralisme ». Commission européenne, DG I, Rapport annuel Tacis 1994, COM (95) 349 final, Bruxelles, 18/07/1995, p. 12.

29. Le programme Initiative de Productivité organise des stages de formation en Europe occidentale pour des cadres d'entreprises étatiques russes. Le programme ESSN (European Senior Service Network) favorise les échanges entre les cadres retraités des entreprises européennes et leurs homologues russes (entreprises privatisées).

30. C'est le cas du programme Mercure, destiné à accroître la coopération entre les chambres de commerce des États membres de la Communauté et leurs homologues russes.

31. Le programme Tempus (Trans-European Scheme for Higher Education) favorise les échanges d'étudiants et les partenariats entre universités européennes et russes.

32. Commission européenne, DG IA, Contract information. Budget 1998, juin, Bruxelles, juin 1998, p. 13-14.

33. Article 56 de l'accord de partenariat et de coopération, op. cit.

34. Bulletin de l'Union européenne, $\mathrm{n}^{\circ} 1-2,2000$, point 1.6.102. Le programme d'action 2000 prévoit des projets visant à renforcer l'indépendance des médias, la tolérance interethnique, le renforcement de l'État de droit et la défense des droits individuels.

\section{RÉSUMÉS}

Laure Delcour montre combien il est difficile d'évaluer d'une façon claire et positive les programmes TACIS qui offrent aux pays voisins une base politique et économique. Elle montre combien lesdits schémas de développement élaborés dans un autre contexte sont appliqués sans le feedback nécessaire pour les adapter efficacement et de façon adéquate. Les résultats sont généralement vagues et diffus sur une longue période de temps. Les instruments sont perçus comme de simples outils économiques et politiques au service des intérêts européens, si l'on considère le manque de connaissance du contexte dans les pays récipiendaires de ces aides pensées pour d'autres situations.

Laure Delcour. Impact of Tacis programme in the 1990ies : appreciation attempt 
Laure Delcour also shows how difficult it is to evaluate in a clear and positive manner the TACIS programmes which are offered to neighbouring countries on an economic and political basis. She emphasizes as well how such development schemes said to be of technical assistance and tried elsewhere are applied without the necessary feedback to adapt them efficiently and adequately. Results are generally vague and spread over a long period of time. Instruments are often perceived as simple economic and political tools serving European interests, considering the general lack of understanding of the context within the countries which are at the receiving end of this help thought out for other countries.

\section{AUTEUR}

\section{LAURE DELCOUR}

Docteur en science politique, maître de conférences à l'Institut d'Études Politiques de Paris, lauredelcour@aol.com 